Chaos, Solitons and Fractals. Vol.67. (2014) 26-37.

\title{
Flow of Fractal Fluid in Pipes: Non-Integer Dimensional Space Approach
}

\author{
Vasily E. Tarasov \\ Skobeltsyn Institute of Nuclear Physics, \\ Lomonosov Moscow State University, Moscow 119991, Russia \\ E-mail: tarasov@theory.sinp.msu.ru
}

\begin{abstract}
Using a generalization of vector calculus for the case of non-integer dimensional space we consider a Poiseuille flow of an incompressible viscous fractal fluid in the pipe. Fractal fluid is described as a continuum in non-integer dimensional space. A generalization of the Navier-Stokes equations for non-integer dimensional space, its solution for steady flow of fractal fluid in a pipe and corresponding fractal fluid discharge are suggested.
\end{abstract}

\section{Introduction}

A cornerstone of fractal fluids is the non-integer dimension [1, 2, 3]. The mass of fractal fluid satisfies a power law relation $M \sim R^{D}$, where $M$ is the mass of the ball region with radius $R$, and $D$ is the mass dimension [4]. Fractal fluid can be described by four different approaches: (1) Using the methods of "Analysis on fractals" [5, 6, 7, 8, 9, 10] it is possible to describe fractal media; (2) An application of fractional-differential continuum models suggested in [11, 12], and then developed in [13, 14, 15, 16, 17, 18], where so-called local fractional derivatives [19] are used; (3) Applying fractional-integral continuum models suggested in [20, 21, 22, 23, 4] (see also [24]-[28] and [29]-[32]), where integrations of non-integer orders and a notion of density of states [4] are used; (4) Fractal media can be described by using the theory of integration and differentiation for a non-integer dimensional space [33, 34, 35] (see also [72, 73].

Let us note that main difference of the continuum models with non-integer dimensional spaces form the fractional continuum models suggested in [20, 21, 22, 23, 4] may be reduced to the following. (a) Arbitrariness in the choice of the numerical factor in the density of states is fixed by the equation of the volume of non-integer dimensional ball region. (b) In the fractional continuum models suggested in [20, 21, 4], the differentiations are integer orders whereas the integrations are non-integer orders. In the continuum models with non-integer dimensional spaces the integrations and differentiations are defined for the spaces with noninteger dimensions.

In this paper, we consider approach based on the non-integer dimensional space. The power law $M \sim R^{D}$ can be naturally derived by using the integrations in non-integer dimensional space [33], where the mass dimension of fractal fluid is connected with the dimension of this 
space. A vector calculus for non-integer dimensional space proposed in this paper allows us to use continuum models with non-integer dimensional spaces to describe for fractal fluids. This is due to the fact that although the non-integer dimension does not reflect completely the geometric properties of the fractal media, it nevertheless permits a number of important conclusions about the behavior of fractal structures. Therefore continuum models with noninteger dimensional spaces can be successfully used to describe fractal fluids.

Integration over non-integer dimensional spaces are actively used in the theory of critical phenomena and phase transitions in statistical physics [36, 37], and in the dimensional regularization of ultraviolet divergences in quantum field theory [38, 39, 33]. The axioms for integrations in non-integer dimensional space are proposed in [40, 34] and this type of integration is considered in the book by Collins [33] for rotationally covariant functions. In the paper [34] a mathematical basis of integration on non-integer dimensional space is given. Stillinger [34] suggested a generalization of the Laplace operator for non-integer dimensional spaces also. Using a product measure approach, the Stillinger's methods [34] has been generalized by Palmer and Stavrinou [35] for multiple variables case with different degrees of confinement in orthogonal directions. The scalar Laplace operators suggested by Stillinger in [34] and Palmer, Stavrinou in [35] for non-integer dimensional spaces, have successfully been used for effective descriptions in physics and mechanics. The Stillinger's form of Laplacian for the Schrödinger equation in non-integer dimensional space is used by He [41, 42, 43] to describe a measure of the anisotropy and confinement by the effective non-integer dimensions. Quantum mechanical models with non-integer (fractional) dimensional space have been discussed in [34, 35, 44, 45, 46, 47, 48] and [49, 50, 51, 52. Recent progress in non-integer dimensional space approach also includes description of the fractional diffusion processes in non-integer dimensional space in [53], and the electromagnetic fields in non-integer dimensional space in [54, 55, 56] and [57, 58, 59, 60, 61.

Unfortunately, [34, 35] proposed only the second order differential operators for scalar fields in the form of the scalar Laplacian in the non-integer dimensional space. A generalization of the vector Laplacian [62] for the non-integer dimensional space is not suggested in [34, 35. The first order operators such as gradient, divergence, curl operators are not considered in [34, 35] also. In the work [61] the gradient, divergence, and curl operators are suggested only as approximations of the square of the Laplace operator. Consideration only the scalar Laplacian in the non-integer dimensional space approach greatly restricts us in application of continuum models with non-integer dimensional spaces for fractal fluids and material. For example, we cannot use the Stillinger's form of Laplacian for vector field $\mathbf{v}(\mathbf{r}, t)$ in hydrodynamics of fractal fluids, in fractal theory of elasticity and thermoelasticity, in electromagnetic theory of fractal media to describe processes in the framework non-integer dimensional space approach.

In this paper, we propose to use a vector calculus for non-integer dimensional space, and we define the first and second orders differential vector operations such as gradient, divergence, the scalar and vector Laplace operators for non-integer dimensional space. In order to derive the vector differential operators in non-integer dimensional space we use the method of analytic continuation in dimension. For simplification we consider rotationally covariant scalar and vector functions that are independent of angles. It allows us to reduce differential equations in 
non-integer dimensional space to ordinary differential equations with respect to $r$. The proposed operators allow us to describe fractal media to describe processes in the framework of continuum models with non-integer dimensional spaces. In this paper we describe a Poiseuille flow of an incompressible viscous fractal fluid in the pipe. A generalization of the Navier-Stokes equation for non-integer dimensional space to describe for fractal fluid are suggested. A solution of this equation for steady flow of fractal fluid in a pipe and corresponding fractal fluid discharge are derived.

\section{Fractal fluids}

A basic characteristic of fractal fluids is the non-integer dimensions such as mass or "particle" dimensions [4]. For fractal fluids the number of particles $N_{D}(W)$ or mass $M_{D}(W)$ in any region $W \subset \mathbb{R}^{3}$ of this fluid increase more slowly than the 3-dimensional volume $V_{3}(W)$ of this region. For the ball region $W$ with radius $R$ in an isotropic fractal fluid, this property can be described by the relation between the number of particles $N_{D}(W)$ in the region $W$ of fractal fluid, and the radius $R$ in the form

$$
N_{D}(W)=N_{0}\left(R / R_{0}\right)^{D}, \quad R / R_{0} \gg 1,
$$

where $R_{0}$ is the characteristic size of fractal fluid such as a minimal scale of self-similarity of a considered fractal fluid. The number $D$ is called the "particle" dimension. It is a measure of how the fluid particles fill the space. The parameter $D$ does not depend on the shape of the region $W$. Therefore fractal fluids can be considered as fluid with non-integer "particle" or mass dimension.

If the fractal fluid consists of particles with identical masses $m_{0}$, then relation (1) gives

$$
M_{D}(W)=M_{0}\left(R / R_{0}\right)^{D}, \quad R / R_{0} \gg 1,
$$

where $M_{0}=m_{0} N_{0}$. In this case, the mass dimension coincides with the "particle" dimension.

As the basic mathematical tool for continuum models of fractal fluids, we propose to use the integration and differentiation in non-integer dimensional spaces. In Section 7, we will show that the power-law relation (2) for an isotropic fractal fluid can be naturally derived by using the integration over non-integer dimensional space, where the space dimension is equal to the mass dimension of fractal fluid.

In order to describe fractal fluid by continuum models with non-integer dimensional spaces, we use the concepts of density of states $c_{3}(D, \mathbf{r})$ that describes how closely packed permitted places (states) in the space $\mathbb{R}^{3}$, where the fractal fluid is distributed. The expression $d V_{D}(\mathbf{r})=$ $c_{3}(D, \mathbf{r}) d V_{3}$ is equal to the number of permitted places (states) between $V_{3}$ and $V_{3}+d V_{3}$ in $\mathbb{R}^{3}$. The notation $d^{D} \mathbf{r}$ also will be used instead of $d V_{D}(\mathbf{r})$. Note that density of states and distribution function are different concepts, and it is impossible to describe all properties of fractal fluids by the distribution function only. 
For fractal fluids, we can use the equation

$$
d N_{D}(W)=n(\mathbf{r}) d V_{D}(\mathbf{r})
$$

where $n(\mathbf{r})$ is a concentration of particles that describes a distribution of number of particles on a set of permitted places (possible states). The density of states is chosen such that $d V_{D}(\mathbf{r})=$ $c_{3}(D, \mathbf{r}) d V_{3}$ describes the number of permitted states in $d V_{3}$.

The form of the function $c_{3}(D, \mathbf{r})$ is defined by symmetries of considered problem and properties of the described fractal fluid. A general property of density of states for fractal fluids is a power-law type of these functions that reflects a scaling property (fractality) of the fractal fluid. To simplify our consideration in this paper we will consider only isotropic fractal fluids with density of states that is independent of angles. In this case, the form of density of states is defined such that $d V_{D}$ is an elementary volume of the non-integer dimensional space.

In the continuum models of fractal fluids, we should work with the dimensionless variables $x / R_{0} \rightarrow x, y / R_{0} \rightarrow x, z / R_{0} \rightarrow x, \mathbf{r} / R_{0} \rightarrow \mathbf{r}$, in order to physical quantities of fractal fluids have correct physical dimensions.

\section{Vector differential operators in non-integer dimensional space}

To derive equations for vector differential operators in non-integer dimensional space, we use equations for the differential operators in the spherical (and cylindrical) coordinates in $\mathbb{R}^{n}$ for arbitrary $n$ to highlight the explicit relations with dimension $n$. Then the vector differential operators for non-integer dimension $D$ can be defined by continuation in dimension from integer

$n$ to non-integer $D$. To simplify we will consider only scalar fields $\varphi$ and vector fields $\mathbf{v}$ that are independent of angles

$$
\varphi(\mathbf{r})=\varphi(r), \quad \mathbf{v}(\mathbf{r})=\mathbf{v}(r)=v_{r} \mathbf{e}_{r},
$$

where $r=|\mathbf{r}|$ is the radial distance, $\mathbf{e}_{r}=\mathbf{r} / r$ is the local orthogonal unit vector in the directions of increasing $r$, and $v_{r}=v_{r}(r)$ is the radial component of $\mathbf{v}$. We will work with rotationally covariant functions only. This simplification is analogous to the simplification for definition of integration over non-integer dimensional space described in Section 4 of the book [33].

\subsection{Vector differential operators for spherical and cylindrical cases}

Using the continuation from integer $n$ to arbitrary non-integer $D$, we can get explicit definitions of differential operators for non-integer dimensional space in the following forms. Note that the same expressions can be obtained by using the integration in non-integer dimensional space and the correspondent Gauss's theorem [72]

Let us define the differential vector operations such as gradient, divergence, the scalar and vector Laplacian for non-integer dimensional space. For simplifications, we assume that the 
vector field $\mathbf{v}=\mathbf{v}(\mathbf{r})$ be radially directed and the scalar and vector fields $\varphi(\mathbf{r}), \mathbf{v}(\mathbf{r})$ are not dependent on the angles.

The divergence in non-integer dimensional space for the vector field $\mathbf{v}=\mathbf{v}(r)$ is

$$
\operatorname{Div}_{r}^{D} \mathbf{v}=\frac{\partial v_{r}}{\partial r}+\frac{D-1}{r} v_{r}
$$

The gradient in non-integer dimensional space for the scalar field $\varphi=\varphi(r)$ is

$$
\operatorname{Grad}_{r}^{D} \varphi=\frac{\partial \varphi}{\partial r} \mathbf{e}_{r}
$$

The scalar Laplacian in non-integer dimensional space for the scalar field $\varphi=\varphi(r)$ is

$$
{ }^{S} \Delta_{r}^{D} \varphi=\operatorname{Div}_{r}^{D} \operatorname{Grad}_{r}^{D} \varphi=\frac{\partial^{2} \varphi}{\partial r^{2}}+\frac{D-1}{r} \frac{\partial \varphi}{\partial r} .
$$

The vector Laplacian in non-integer dimensional space for the vector field $\mathbf{v}=v(r) \mathbf{e}_{r}$ is

$$
{ }^{V} \Delta_{r}^{D} \mathbf{v}=\operatorname{Grad}_{r}^{D} \operatorname{Div}_{r}^{D} \mathbf{v}=\left(\frac{\partial^{2} v_{r}}{\partial r^{2}}+\frac{D-1}{r} \frac{\partial v_{r}}{\partial r}-\frac{D-1}{r^{2}} v_{r}\right) \mathbf{e}_{r} .
$$

If $D=n$, equations (44-7) give the well-known formulas for integer dimensional space $\mathbb{R}^{n}$.

Let us consider a case of axial symmetry of the fluid, where the fields $\varphi(r)$ and $\mathbf{v}(r)=v_{r}(r) \mathbf{e}_{r}$ are also axially symmetric. We will direct the $Z$-axis along the axis of symmetry. Therefore we use a cylindrical coordinate system.

The divergence in non-integer dimensional space for the vector field $\mathbf{v}=\mathbf{v}(r)$ is

$$
\operatorname{Div}_{r}^{D} \mathbf{v}=\frac{\partial v_{r}}{\partial r}+\frac{D-2}{r} v_{r}
$$

The gradient in non-integer dimensional space for the scalar field $\varphi=\varphi(r)$ is

$$
\operatorname{Grad}_{r}^{D} \varphi=\frac{\partial \varphi}{\partial r} \mathbf{e}_{r}
$$

The scalar Laplacian in non-integer dimensional space for the scalar field $\varphi=\varphi(r)$ is

$$
{ }^{S} \Delta_{r}^{D} \varphi=\frac{\partial^{2} \varphi}{\partial r^{2}}+\frac{D-2}{r} \frac{\partial \varphi}{\partial r} .
$$

The vector Laplacian in non-integer dimensional space for the vector field $\mathbf{v}=v(r) \mathbf{e}_{r}$ is

$$
{ }^{V} \Delta_{r}^{D} \mathbf{v}=\left(\frac{\partial^{2} v_{r}}{\partial r^{2}}+\frac{D-2}{r} \frac{\partial v_{r}}{\partial r}-\frac{D-2}{r^{2}} v_{r}\right) \mathbf{e}_{r}
$$


Equations (8,11) can be easy generalized for the case $\varphi=\varphi(r, z)$ and $\mathbf{v}(r, z)=v_{r}(r, z) \mathbf{e}_{r}+$ $v_{r}(r, z) \mathbf{e}_{z}$. In this case the curl operator for $\mathbf{v}(r, z)$ is different from zero, and

$$
\operatorname{Curl}_{r}^{D} \mathbf{v}=\left(\frac{\partial v_{r}}{\partial z}-\frac{\partial v_{z}}{\partial r}\right) \mathbf{e}_{\theta}
$$

For $D=3$ equations (41) - (11) and (12) give the well-known expressions for the gradient, divergence, curl operator, scalar and vector Laplacian operators

The suggested operators for $0<D<3$ allow us to reduce $D$-dimensional vector differentiations (44) - (17) and (8) - (11) to derivatives with respect to $r=|\mathbf{r}|$. It allows us to reduce partial differential equations for fields in non-integer dimensional space to ordinary differential equations with respect to $r$.

\subsection{Stillinger's Laplacian for non-integer dimensional space}

For a function $\varphi=\varphi(r, \theta)$ of radial distance $r$ and related angle $\theta$ measured relative to an axis passing through the origin, the scalar Laplacian in a non-integer dimensional space proposed by Stillinger [34] is

$$
{ }^{S t} \Delta^{D}=\frac{1}{r^{D-1}} \frac{\partial}{\partial r}\left(r^{D-1} \frac{\partial}{\partial r}\right)+\frac{1}{r^{2} \sin ^{D-2} \theta} \frac{\partial}{\partial \theta}\left(\sin ^{D-2} \theta \frac{\partial}{\partial \theta}\right),
$$

where $D$ is the dimension of space $(0<D<3)$, and the variables $r \geq 0,0 \leq \theta \leq \pi$. Note that $\left({ }^{S t} \Delta^{D}\right)^{2} \neq{ }^{S t} \Delta^{2 D}$. If the function depends on radial distance $r$ only $(\varphi=\varphi(r))$, then

$$
{ }^{S t} \Delta^{D} \varphi(r)=\frac{1}{r^{D-1}} \frac{\partial}{\partial r}\left(r^{D-1} \frac{\partial \varphi(r)}{\partial r}\right)=\frac{\partial^{2} \varphi(r)}{\partial r^{2}}+\frac{D-1}{r} \frac{\partial \varphi(r)}{\partial r} .
$$

It is easy to see that the Stillinger's form of Laplacian ${ }^{S t} \Delta^{D}$ for radial scalar functions $\varphi(\mathbf{r})=$ $\varphi(r)$ coincides with the scalar Laplacian ${ }^{S} \Delta_{r}^{D}$ defined by (6) $)$ i.e.,

$$
{ }^{S t} \Delta^{D} \varphi(r)={ }^{S} \Delta^{D} \varphi(r) .
$$

The Stillinger's Laplacian can be applied for scalar fields only. It cannot be used to describe vector fields $\mathbf{v}=v_{r}(r) \mathbf{e}_{r}$ because this Laplacian for $D=3$ is not equal to the usual vector Laplacian for $\mathbb{R}^{3}$,

$$
{ }^{S t} \Delta^{3} \mathbf{v}(r) \neq \Delta \mathbf{v}(r)=\left(\frac{\partial^{2} v_{r}}{\partial r^{2}}+\frac{2}{r} \frac{\partial v_{r}}{\partial r}-\frac{2}{r^{2}} v_{r}\right) \mathbf{e}_{r}
$$

The gradient, divergence, curl operator and vector Laplacian are not considered by Stillinger in paper [34]. 


\subsection{Differential operators for $d \neq D-1$}

Let us consider a ball region $B_{D}$ in the fractal fluids with the boundary $S_{d}=\partial B_{D}$ with dimensions

$$
\operatorname{dim}\left(B_{D}\right)=D, \quad \operatorname{dim}\left(S_{d}\right)=d .
$$

Equations (41) - (11) define differential operators for spaces with non-integer dimension $D$ and boundary dimensions $d=D-1$. In general, the dimension $D$ of the region of a fractal fluid and the dimension $d$ of boundary of this region are not related by the equation $d=D-1$, i.e.,

$$
\operatorname{dim}\left(\partial B_{D}\right) \neq D-1
$$

Using the integration in non-integer dimensional space and the correspondent Gauss's theorem, we can define the divergence for the case $d \neq D-1$ by the equation

$$
\operatorname{Div}_{r}^{D, d} \mathbf{v}=\frac{\pi^{(d+1-D) / 2} \Gamma(D / 2)}{\Gamma((d+1) / 2)}\left(\frac{1}{r^{D-1-d}} \frac{\partial v_{r}(r)}{\partial r}+\frac{d}{r^{D-d}} v_{r}(r)\right) .
$$

For $d=D-1$, we get (41). We can define the parameter

$$
\alpha_{r}=D-d,
$$

that can be interpreted as a dimension of fractal fluid along the radial direction $\mathbf{e}_{r}$. Using (20), equation (19) can be rewritten in the form

$$
\operatorname{Div}_{r}^{D, d} \mathbf{v}=\pi^{\left(1-\alpha_{r}\right) / 2} \frac{\Gamma\left(\left(d+\alpha_{r}\right) / 2\right)}{\Gamma((d+1) / 2)}\left(\frac{1}{r^{\alpha_{r}-1}} \frac{\partial v_{r}(r)}{\partial r}+\frac{d}{r^{\alpha_{r}}} v_{r}(r)\right) .
$$

This is divergence operator for non-integer boundary dimension $d \neq D-1$. For $\alpha_{r}=1$, we have $d=D-1$, and then equations (19), (21) give (4).

The gradient for the scalar field $\varphi(\mathbf{r})=\varphi(r)$ and the radial dimension $\alpha_{r} \neq 1$ is defined by

$$
\operatorname{Grad}_{r}^{D, d} \varphi=\frac{\Gamma\left(\alpha_{r} / 2\right)}{\pi^{\alpha_{r} / 2} r^{\alpha_{r}-1}} \frac{\partial \varphi(r)}{\partial r} \mathbf{e}_{r}
$$

For $\alpha_{r}=1$, equation (22) gives (15).

Using the operators (22) and (19) for the fields $\varphi=\varphi(r)$ and $\mathbf{v}=v(r) \mathbf{e}_{r}$, we can get the scalar and vector Laplace operators for the case $d \neq D-1$ by

$$
{ }^{S} \Delta_{r}^{D, d} \varphi=\operatorname{Div}_{r}^{D, d} \operatorname{Grad}_{r}^{D, d} \varphi, \quad{ }^{V} \Delta_{r}^{D, d} \mathbf{v}=\operatorname{Grad}_{r}^{D, d} \operatorname{Div}_{r}^{D, d} \mathbf{v} .
$$

The scalar Laplacian for $d \neq D-1$ for the field $\varphi=\varphi(r)$ is

$$
{ }^{S} \Delta_{r}^{D, d} \varphi=A\left(d, \alpha_{r}\right)\left(\frac{1}{r^{2 \alpha_{r}-2}} \frac{\partial^{2} \varphi}{\partial r^{2}}+\frac{d+1-\alpha_{r}}{r^{2 \alpha_{r}-1}} \frac{\partial \varphi}{\partial r}\right)
$$


where

$$
A\left(d, \alpha_{r}\right)=\frac{\Gamma\left(\left(d+\alpha_{r}\right) / 2\right) \Gamma\left(\alpha_{r} / 2\right)}{\pi^{\alpha_{r}-1 / 2} \Gamma((d+1) / 2)} .
$$

The vector Laplacian for $d \neq D-1$ for the field $\mathbf{v}=v(r) \mathbf{e}_{r}$ is

$$
{ }^{V} \Delta_{r}^{D, d} \mathbf{v}=A\left(d, \alpha_{r}\right)\left(\frac{1}{r^{2 \alpha_{r}-2}} \frac{\partial^{2} v_{r}}{\partial r^{2}}+\frac{d+1-\alpha_{r}}{r^{2 \alpha_{r}-1}} \frac{\partial v_{r}}{\partial r}-\frac{d \alpha_{r}}{r^{2 \alpha_{r}}} v_{r}\right) \mathbf{e}_{r}
$$

The vector differential operators (22), (19), (24) and (26) allow us to describe complex fractal fluids with the boundary dimensions $d \neq D-1$ by continuum models with non-integer dimensional spaces.

\section{Navier-Stokes equations in non-integer dimensional space for fractal fluid}

A motion of an incompressible viscous fractal fluid in the framework of continuum model with non-integer dimensional space is described by the equations

$$
\begin{gathered}
\operatorname{Div}_{r}^{D} \mathbf{v}=0, \\
\frac{d \mathbf{v}}{d t}=\mathbf{f}-\frac{1}{\rho} \operatorname{Grad}_{r}^{D} p+\nu^{V} \Delta_{r}^{D} \mathbf{v},
\end{gathered}
$$

where $\mathbf{f}$ is the vector field of mass forces, $\nu$ is the kinematic viscosity is the ratio of the dynamic viscosity $\mu$ to the density of the fluid $\rho$, and $d / d t$ is the material derivative

$$
\frac{d \mathbf{v}}{d t}=\frac{\partial \mathbf{v}}{\partial t}+\left(\mathbf{v}, \operatorname{Grad}_{r}^{D} \mathbf{v}\right)
$$

In equations (27)-(29) the gradient $\operatorname{Grad}_{r}^{D}$, the divergence $\operatorname{Div}_{r}^{D}$, and the vector Laplacian ${ }^{V} \Delta_{r}^{D}$ are defined by equations (5), (4), (17) for spherical symmetry. For cylindrical symmetry, these operators are defined by equations (9), (8), (11).

If the dimension $D$ of the region of a fractal fluid and the dimension $d$ of boundary of this region are not related by the relation $d=D-1$, i.e., $\alpha_{r}=D-d \neq 1$, then we should use the equations

$$
\begin{gathered}
\operatorname{Div}_{r}^{D, d} \mathbf{v}=0 \\
\frac{d \mathbf{v}}{d t}=\mathbf{f}-\frac{1}{\rho} \operatorname{Grad}_{r}^{D, d} p+\nu^{V} \Delta_{r}^{D, d} \mathbf{v} \\
\frac{d \mathbf{v}}{d t}=\frac{\partial \mathbf{v}}{\partial t}+\left(\mathbf{v}, \operatorname{Grad}_{r}^{D, d} \mathbf{v}\right)
\end{gathered}
$$

where the gradient $\operatorname{Grad}_{r}^{D, d}$, the divergence $\operatorname{Div}_{r}^{D, d}$, and the vector Laplacian ${ }^{V} \Delta_{r}^{D, d}$ are defined by equations (22), (21), (26). 
Equations (28) and (31) can be called the Navier-Stokes equations for non-integer dimensional space.

It is convenient to work in the dimensionless space variables $x / R_{0} \rightarrow x, y / R_{0} \rightarrow x, z / R_{0} \rightarrow$ $x, r / R_{0} \rightarrow r$, that yields dimensionless integration and dimensionless differentiation in noninteger dimensional space. Here $R_{0}$ is the characteristic size of a fractal fluid, which is always finite. For example, $R_{0}$ can be the minimal scale of self-similarity of a considered fractal fluid. Then the density is properly scaled such that the mass $Q$ of fractal fluid and the fields $\mathbf{v}, p, f$ have correct physical dimensions.

The Navier-Stokes equations (28) and (31) describe dynamics of fractal fluids in the framework of continuum models with non-integer dimensional spaces. These equations allow us to describe the isotropic fractal fluid only when the presence of spherical or cylindrical symmetry.

Equations (28) and (31) can be used, when the fields $p, \mathbf{v}, \mathbf{v}$ have the form $p=p(r)$ and $\left.\mathbf{v}=v_{r}(r) \mathbf{e}_{r}, \mathbf{f}\right)=f_{r}(r) \mathbf{e}_{r}$ does not depend on the angles.

As an example of application of the Navier-Stokes equations (28) and (31), we consider a steady flow of fractal fluid in a pipe, and fractal fluid discharge in the next sections. In the next sections, we derive the Poiseuille equation for fractal fluids from the Navier-Stokes equations (28) and (31). Equations can be used for any other problems of hydrostatics and hydrodynamics of fractal fluids within the case of spherical and cylindrical symmetries

To consider anisotropic fractal fluids, and problems without spherical and cylindrical symmetries, we cannot use the Navier-Stokes equations (28) and (31). In this case, we should apply a product measure approach [73]. The product measure approach to describe fractal properties of space-time has been considered in [63. The product measure approach for the fractional spaces has been suggested in [64, 65], where fractional phase space is considered with its interpretation as a non-integer (fractional) dimensional space. For non-integer dimensional spaces the product measure approach is suggested in [35], where each orthogonal coordinates has own dimension. The product measure approach for the fractional-integral continuum models has been considered in [25, 26, 27, 28].

\section{Steady flow of fractal fluid in a pipe}

In this section, we derive the Poiseuille equation for fractal fluids from the Navier-Stokes equations (28). Let us consider a simple problem of motion of an incompressible viscous fractal fluid. Using the continuum models with non-integer dimensional space, we describe a steady flow of fractal fluid in a pipe with circular cross-section. We take the axis of the pipe as the $X$-axis. The velocity for laminar of fractal fluid is along the $X$-axis at all points, and is a function of $r$ only

$$
\mathbf{v}=\mathbf{v}(r)=v_{x}(r) \mathbf{e}_{x} .
$$

The equation of continuity is satisfied identically. The components of the Navier-Stokes equation for $Y$-axis and $Z$-axis give that the pressure is constant over the cross-section of the pipe. 
We shall solve the equation for a pipe with circular cross-section. Taking the origin at the center of the circle we can use cylindrical symmetry $\mathbf{v}=\mathbf{v}(r)=v_{x}(r) \mathbf{e}_{x}$. Using the Navier-Stokes equations (28), we have

$$
{ }^{S} \Delta_{r}^{D} v_{x}(r)=\frac{1}{\mu} \frac{d p}{d x}
$$

where $\mu=\rho \nu$, and $d p / d x$ is a constant. The pressure gradient $d p / d x$ may be written $-\Delta p / l$, where $\Delta p$ is the pressure difference between the ends of the pipe and $l$ is its length.

Using the scalar Laplacian for non-integer dimensional space the Navier-Stokes equation (34) takes the form

$$
\frac{\partial^{2} v_{x}(r)}{\partial r^{2}}+\frac{D-2}{r} \frac{\partial v_{x}}{\partial r}-\frac{1}{\mu} \frac{d p}{d x}=0 .
$$

For $1<D<3$ and $0<D<1$, the general solution of (35) is

$$
v_{x}(r)=C_{1} r^{3-D}+C_{2}+\frac{1}{2(D-1) \mu} \frac{d p}{d x} r^{2} \quad(0<D<3, \quad D \neq 1) .
$$

For $D=3$, we have

$$
v_{x}(r)=C_{1} \ln (r)+C_{2}+\frac{1}{4 \mu} \frac{d p}{d x} r^{2} .
$$

For $D=1$, we get the general solution

$$
v_{x}(r)=C_{1} r^{2}+C_{2}+\frac{1}{4 \mu} \frac{d p}{d x} r^{2}(2 \ln (r)-1) .
$$

It should be noted that dimensions $D=1$ of the fractal fluid do not correspond to the distribution of particles along the line. The fractal media with $D=1$ describe a distribution of fluid particles in 3-dimensional space such that the mass dimension of the distribution is equal to $D=1$.

Let us determine a flow of fractal fluid in a pipe of annular cross-section with the internal radius $R_{1}$ and external radius $R_{2}$. The constants $C_{1}$ and $C_{2}$ in the general solution (36) are determined from the boundary conditions

$$
v_{x}\left(R_{1}\right)=v_{x}\left(R_{2}\right)=0 .
$$

Using (36), these conditions have the form

$$
\begin{aligned}
& C_{1} R_{1}^{3-D}+C_{2}+\frac{1}{2(D-1) \mu} \frac{d p}{d x} R_{1}^{2}=0, \\
& C_{1} R_{2}^{3-D}+C_{2}+\frac{1}{2(D-1) \mu} \frac{d p}{d x} R_{2}^{2}=0 .
\end{aligned}
$$


Then the constants are

$$
\begin{gathered}
C_{1}=\frac{1}{2(D-1) \mu} \frac{d p}{d x} \frac{R_{2}^{2}-R_{1}^{2}}{R_{1}^{3-D}-R_{2}^{3-D},} \\
C_{2}=\frac{1}{2(D-1) \mu} \frac{d p}{d x} \frac{R_{1}^{3-D} R_{2}^{2}-R_{2}^{3-D} R_{1}^{2}}{R_{1}^{3-D}-R_{2}^{3-D}} .
\end{gathered}
$$

Substitution of (42) and (43) into (36) gives

$$
v_{x}(r)=\frac{1}{2(D-1) \mu} \frac{d p}{d x}\left(\frac{R_{2}^{2}-R_{1}^{2}}{R_{1}^{3-D}-R_{2}^{3-D}} r^{3-D}+\frac{R_{1}^{3-D} R_{2}^{2}-R_{2}^{3-D} R_{1}^{2}}{R_{1}^{3-D}-R_{2}^{3-D}}+r^{2}\right),
$$

where $0<D<1$ and $1<D<3$.

Using the variables

$$
x=\frac{R_{2}}{R_{1}}, \quad y=\frac{r}{R_{1}},
$$

equation (44) can be represented in the form

$$
v(x, y)=\frac{R_{1}^{2}}{2(D-1) \mu} \frac{d p}{d x}\left(-\frac{1-x^{2}}{1-x^{3-D}} y^{3-D}+\frac{x^{2}-x^{3-D}}{1-x^{3-D}}+y^{2}\right),
$$

To demonstrate some properties of the velocity $v_{x}(r)$ defined by (44), we can visualize the function (45), for $x \in[1 ; 100], y \in[1 ; 100]$ and different values of dimensions $D=2.9, D=2.7$, $D=2.0, D=1.1$. The plots of function (45) are presented by Figures $1-4$, where $\mu=1, R_{1}=1$ and $d p / d x=-1$.

The flow in a pipe of annular cross-section with the radius $R$, i.e. $R_{1}=0$ and $R_{2}=R$, we have

$$
v_{x}(r)=-\frac{1}{2(D-1) \mu} \frac{d p}{d x} R^{2}\left(\left(\frac{r}{R}\right)^{3-D}-\left(\frac{r}{R}\right)^{2}\right) \quad(0<D \leq 3, \quad D \neq 1) .
$$

Equation (46) can be called the Poiseuille equation for flow of fractal fluid. For the case of non-fractal fluid $(D=3)$, equation (46) gives the well-known Poiseuille equation

$$
v_{x}(r)=-\frac{1}{4 \mu} \frac{d p}{d x} R^{2}\left(1-\left(\frac{r}{R}\right)^{2}\right) .
$$

Thus the velocity distribution across the pipe is parabolic for the non-fractal fluids. For the fractal fluids, we have non-integer power-law (46).

Note that suggested Poiseuille equation for fractal fluids, which are derived from the proposed Navier-Stokes equation for non-integer dimensional space can be used only to describe flow of fractal fluid in pipes. To describe flow of fractal fluid between parallel planes, we should define new differential operators and the Navier-Stokes equations (28) and (31), should be modified by using the product measure approach [63, 35, 64, 65]. 


\section{$6 \quad$ Fractal fluid with $\alpha_{r} \neq 1$}

Let us derive the Poiseuille equation for fractal fluids from the Navier-Stokes equations (31). The Navier-Stokes equations for fractal fluid with $\alpha_{r}=D-d \neq 1$ has the form

$$
A\left(d_{x}, \alpha_{r}\right)\left(\frac{1}{r^{2 \alpha_{r}-2}} \frac{\partial^{2} v_{x}(r)}{\partial r^{2}}+\frac{d_{x}+1-\alpha_{r}}{r^{2 \alpha_{r}-1}} \frac{\partial v_{x}(r)}{\partial r}\right)-\frac{1}{\mu} \frac{d p}{d x}=0
$$

where $A\left(d_{x}, \alpha_{r}\right)$ is defined by (25),$d_{x}=d-\alpha_{x}$, and $\alpha_{x}$ is dimension along the $X$-axis. Using $v_{x}(r)$ as an effective scalar field $\varphi_{\text {eff }}(r)=v_{x}(r)$, we can apply equations (22), (21) and (24) where $D \rightarrow D_{x}=D-\alpha_{x}$ and $d \rightarrow d_{x}=d-\alpha_{x}$ to get (48). Equation (48) with $\alpha_{r}=\alpha_{x}=1$ gives (35).

For $1<D<3$ and $0<D<1$, the general solution of (48) is

$$
v_{x}(r)=C_{1} r^{\alpha_{r}-d_{x}}+C_{2}+\frac{1}{2\left(d_{x}+\alpha_{r}\right) \alpha_{r} A\left(d_{x}, \alpha_{r}\right) \mu} \frac{d p}{d x} r^{2 \alpha_{r}} \quad(0<D<3, \quad D \neq 1) .
$$

For $\alpha_{r}=\alpha_{x}=1$ equation (49) gives (36). The constants $C_{1}$ and $C_{2}$ in the general solution (49) are determined by the boundary conditions

$$
v_{x}\left(R_{1}\right)=v_{x}\left(R_{2}\right)=0
$$

These conditions give the equations

$$
\begin{aligned}
& C_{1} R_{1}^{\alpha_{r}-d_{x}}+C_{2}+\frac{1}{2\left(d_{x}+\alpha_{r}\right) \alpha_{r} A\left(d_{x}, \alpha_{r}\right) \mu} \frac{d p}{d x} R_{1}^{2 \alpha_{r}}=0, \\
& C_{1} R_{2}^{\alpha_{r}-d_{x}}+C_{2}+\frac{1}{2\left(d_{x}+\alpha_{r}\right) \alpha_{r} A\left(d_{x}, \alpha_{r}\right) \mu} \frac{d p}{d x} R_{2}^{2 \alpha_{r}}=0 .
\end{aligned}
$$

Then the coefficients are

$$
\begin{gathered}
C_{1}=-\frac{1}{2\left(d_{x}+\alpha_{r}\right) \alpha_{r} A\left(d_{x}, \alpha_{r}\right) \mu} \frac{d p}{d x} \frac{R_{1}^{2 \alpha_{r}}-R_{2}^{2 \alpha_{r}}}{R_{1}^{\alpha_{r}-d_{x}}-R_{2}^{\alpha_{r}-d_{x}}} \\
C_{3}=-\frac{1}{2\left(d_{x}+\alpha_{r}\right) \alpha_{r} A\left(d_{x}, \alpha_{r}\right) \mu} \frac{d p}{d x} \frac{R_{2}^{2 \alpha_{r}} R_{1}^{\alpha_{r}-d_{x}}-R_{1}^{2 \alpha_{r}} R_{2}^{\alpha_{r}-d_{x}}}{R_{1}^{\alpha_{r}-d_{x}}-R_{2}^{\alpha_{r}-d_{x}}},
\end{gathered}
$$

Substitution of (53) and (154) into (49) gives

$$
\begin{gathered}
v_{x}(r)=-\frac{1}{2\left(d_{x}+\alpha_{r}\right) \alpha_{r} A\left(d_{x}, \alpha_{r}\right) \mu} \frac{d p}{d x}\left(\frac{R_{1}^{2 \alpha_{r}}-R_{2}^{2 \alpha_{r}}}{R_{1}^{\alpha_{r}-d_{x}}-R_{2}^{\alpha_{r}-d_{x}}} r^{\alpha_{r}-d_{x}}+\right. \\
\left.+\frac{R_{2}^{2 \alpha_{r}} R_{1}^{\alpha_{r}-d_{x}}-R_{1}^{2 \alpha_{r}} R_{2}^{\alpha_{r}-d_{x}}}{R_{1}^{\alpha_{r}-d_{x}}-R_{2}^{\alpha_{r}-d_{x}}}-r^{2 \alpha_{r}}\right)
\end{gathered}
$$


for $0<D<3$, where $D \neq 1$.

If $R_{1}=0$ and $R_{2}=R$, then equation (55) has the form

$$
v_{x}(r)=-\frac{1}{2\left(d_{x}+\alpha_{r}\right) \alpha_{r} A\left(d_{x}, \alpha_{r}\right) \mu} \frac{d p}{d x} R^{2 \alpha_{r}}\left(\left(\frac{r}{R}\right)^{\alpha_{r}-d_{x}}-\left(\frac{r}{R}\right)^{2 \alpha_{r}}\right) .
$$

For $\alpha_{r}=\alpha_{x}=1$ equation (56) gives (46). The cases $\alpha_{r}<1$ and/or $\alpha_{x}<1$ corresponds to fractal fluid.

We can assume that $\alpha_{x}>1$ can be used to describe fractal turbulent flow in pipe. This assumption is based on the fact that trajectories of the fluid particles are fractal curve, then $\alpha_{x}>1$ (for example, the Koch curve with $\alpha_{x}=\ln (4) / \ln (3) \approx 1.262$ ).

\section{$7 \quad$ Fractal fluid discharge}

In general, fractal fluids cannot be considered as a fluid on fractal. Real fractal fluids have a characteristic smallest length scale such as the radius, $R_{0}$, of a particle (for example, an atom or molecule). In real fluids the fractal structure cannot be observed on all scales but only those for which $R>R_{0}$, where $R_{0}$ is the characteristic scale of the particles. The concept of non-integer mass dimension of fractal fluid is based on the idea of how the mass of a fluid region scales with the region size, if we assume unchanged density. For many cases, we can write the asymptotic form for the relation between the mass $M_{D}(W)$ of a ball region $W$ of fluid, and the radius $R$ containing this mass as follows:

$$
M_{D}(W)=M_{0}\left(\frac{R}{R_{0}}\right)^{D}
$$

for $R / R_{0} \gg 1$. The constant $M_{0}$ depends on how the spheres of radius $R_{0}$ are packed. The parameter $D$, which is interpreted as a dimension, does not depend on the shape of the region $W$, or on whether the packing of spheres of radius $R_{0}$ is close packing, a random packing or a porous packing with a uniform distribution of holes. The non-integer mass dimension $D$ of fractal fluid is a measure of how the fluid fills the integer $n$-dimensional Euclidean space it occupies. Note that the fact that a fluid is random or contains cavities does not necessarily imply that the fluid is fractal.

Using the non-integer dimensional space approach, we can calculate the mass of fractal homogeneous fluids. Scaling law (57) is obtained naturally in the framework of this approach. We can use the integration in a non-integer dimensional space [34] that is described by the equation

$$
\int_{R^{D}} d^{D} \mathbf{r} \varphi(\mathbf{r})=\frac{2 \pi^{(D-1) / 2}}{\Gamma((D-1) / 2)} \int_{0}^{\infty} d r r^{D-1} \int_{0}^{\pi} d \theta \sin ^{D-2} \theta \varphi(r, \theta),
$$

where $d^{D} \mathbf{r}$ represent the volume element in the non-integer dimensional space. Using (58) with $\varphi(r, \theta)=1$, and

$$
\int_{0}^{\pi} d \theta \sin ^{D-2} \theta=\frac{\pi^{1 / 2} \Gamma(D / 2-1)}{\Gamma(D / 2)}
$$


we get the volume of $D$-dimensional ball with radius $R$ in the form

$$
V_{D}=\frac{\pi^{D / 2}}{\Gamma(D / 2+1)} R^{D}
$$

The mass of fluid in $W$ is described by the integral

$$
M_{D}(W)=\int_{W} \rho(\mathbf{r}) d^{D} \mathbf{r}
$$

where $\mathbf{r}$ is dimensionless vector variable. For a ball with radius $R$ and constant density $\rho(\mathbf{r})=$ $\rho=$ const, we get

$$
M_{D}(W)=\rho V_{D}=\frac{\pi^{D / 2} \rho}{\Gamma(D / 2+1)} R^{D}
$$

This equation define the mass of the fractal homogeneous ball region of fluid with volume $V_{D}$. For $D=3$, equation (62) gives the well-known equation for mass of non-fractal ball region $M_{3}=(4 \rho \pi / 3) R^{3}$ because $\Gamma(3 / 2)=\sqrt{\pi} / 2$ and $\Gamma(z+1)=z \Gamma(z)$.

Let us determine the mass $Q$ of fluid passing per unit time through any cross-section of the pipe (called the discharge). Not all pipe volume is occupied by fractal fluid. There are areas unoccupied by particles of fractal fluid. In continuum model of fractal fluids, we take into account this fact by using the integration in space with non-integer dimension

$$
Q=\rho \frac{2 \pi^{d / 2}}{\Gamma(d / 2)} \int_{0}^{R} v_{x}(r) r^{d-1} d r
$$

where $d=D-1$ is non-integer dimension of the cross-section, $\rho$ is a constant density, and $v_{x}(r)$ is defined by equation (46). Substitution of (46) into (163) gives

$$
Q=-\frac{\rho \pi^{(D-1) / 2}}{2(D+1) \Gamma((D-1) / 2) \mu} \frac{d p}{d x} R^{D+1} \quad(0<D \leq 3 \quad D \neq 1) .
$$

Note that $\rho$ has physical dimension of mass (for example, kilogram). The mass of fractal fluid is thus proportional to $(D+1)$-power of the radius of the pipe.

For $D=3$, equation (64) gives the well-known equation

$$
Q=-\frac{\rho \pi}{8 \mu} \frac{d p}{d x} R^{4}
$$

The mass of non-fractal fluid is proportional to the fourth power of the radius of the pipe. The dependence of $Q$ on $d p / d x$ and $R$ given by formula (65) was established empirically by $\mathrm{G}$. Hagen in 1839 and J. L. M. Poiseuille in 1840, and theoretically justified by G. G. Stokes in 1845.

Equation (64) can be rewritten in the form

$$
Q=-\frac{\rho \pi}{8 \mu_{e f f}} \frac{d p}{d x} R^{D+1}
$$


with the effective dynamic viscosity

$$
\mu_{e f f}=\frac{D+1}{4} \pi^{(3-D) / 2} \Gamma((D-1) / 2) \mu .
$$

The mass (discharge) of fractal fluid is proportional to the non-integer power $(D+1)<4$ of the radius of the pipe, and the dynamic viscosity is effectively changed.

For $D=3$, equation (67) gives $\mu_{\text {eff }}=\mu$. For $1<D<3$, we have $\mu_{\text {eff }}>\mu$. If $0<D<1$, then $\mu_{\text {eff }}<0$. The effective dynamic viscosity of fractal fluid with $D \in(1 ; 3)$ increases with increasing a deviation of the dimension $D$ from three. We can see an interesting effect of a negative effective dynamic viscosity for fractal fluid with dimension $D \in(0 ; 1)$. The strong fractality of the fluid, which is caused by small dimension, leads to an increased fluid flow compared with conventional medium. This is probably due to an increase in freedom of particles motion for the fractal fluid similar to 3D Cantor dust.

The discharge function $Q=Q(R, D)$ defined by (64) for the different values of dimensions $0<D<3$ and the range $R \in[0,1]$ are present on Figures 5 -8, where $\rho=1, \mu=1$, and $d p / d x=-1$.

\section{Conclusion}

In this paper, we propose a generalization of the Navier-Stokes equations to describe fractal fluids in the framework of continuum models with non-integer dimensional spaces. These equations contain generalized differential vector operators for non-integer dimensional space [72, 73]. As an example of application of the suggested Navier-Stokes equations for fractal fluids, we consider a Poiseuille flow of an incompressible viscous fractal fluid in the pipe. The solution for steady flow of fractal fluid in a pipe and corresponding fractal fluid discharge have been derived.

In this paper fractal fluid is described as a continuum in non-integer dimensional space. We assume that suggested continuum models with non-integer dimensional spaces and the correspondent Navier-Stokes equations for fractal fluids may be important for fractal theory of different type of media.

As the main object for application of the proposed continuum models is a two-component medium, where distribution of one component (gas, liquid, solid) into another component (fluid, gas or empty space) can be characterized by non-integer mass or "particle" dimension. This non-integer dimensional component can be considered as a fractal fluid. One of the possible experimental methods for determining the presence of fractal properties of the two-component medium may be to use labels with radioactive isotopes for particles of component that is assumed a fractal.

A basic idealized model of fractal fluid is a liquid distributed in empty space $\mathbb{R}^{3}$ with noninteger mass dimension $D<3$. In some sense the fractal fluid is considered as a liquid analog of fractal porous solid material. Fractal fluid can also be viewed as a two-phase medium consisting 
of a liquid and a discharged gas instead of empty space, where the liquid is characterized by fractal mass dimension.

As an object of study, we also can consider an emulsion, when both the dispersed and the continuous phase are liquids, and the dispersed phase is fractally distributed in continuous phase. An emulsion that is a mixture of two immiscible liquids, one of which (the dispersed phase) is fractally dispersed in the other (the continuous phase). In this case the dispersed phase can be described as a fractal fluid by suggested continuum models with non-integer dimensional space. The proposed models can be used for a solution that is a homogeneous mixture composed of only one (liquid) phase, where one phase has a fractal dimension. We can consider a fractal distribution of a solute dissolved in a non-fractal solvent, then the solute is considered as a fractal fluid. The solvent that is fractally homogeneously mixed with solute can be considered as a fractal homogeneous fluid. The homogeneity property of the fractal fluid means that two regions $W_{1}$ and $W_{2}$ with the equal volumes $V_{n}\left(W_{1}\right)=V_{n}\left(W_{2}\right)$ have equal number of particles $N_{D}\left(W_{1}\right)=N_{D}\left(W_{2}\right)$. In other words the fractal fluid is called homogeneous if the power law $N_{D}(W) \sim R^{D}$ (or $M_{D}(W) \sim R^{D}$ ) does not depend on the translation of the region $W$.

We can consider a fractal distribution of small solid particles in the suspension. In this case, we have an internal phase (solid) that is fractally distributed through the external phase (fluid) by mechanical agitation.

An object of investigations can be a liquid mixed with a solid particles, where the distribution of these particles in space can be characterized by non-integer mass dimensions, which can be caused by a power law distribution of particles by size or mass.

As a complex medium which may exhibit fractal properties can be considered the blood that is composed of proteins, glucose, mineral ions, hormones, carbon dioxide, blood cells and other particles suspended in water. We assume that the blood as a multi-phase medium can have attributes of a fractal distribution for some blood components including bacteria, viruses and medicinal substances getting into the blood.

We assume that the suggested approach to describe fractal fluids by continuum models with non-integer dimensional spaces may be important for fractal theory of blood flow in cardiovascular system, dynamics of fractal media in hydrologic modeling [66, 67, 68, 69] and it allows to develop the fractal dynamics of multi-phase media [70, 71].

\section{References}

[1] K.F. Falconer, The Geometry of Fractal Sets (Cambridge University Press, 1985).

[2] J. Feder, Fractals (Plenum Press, New York, 1988).

[3] A. Carpinteri, F. Mainardi (Eds.), Fractals and Fractional Calculus in Continuum Mechanics (Springer, New York, 1997).

[4] V.E. Tarasov, Fractional Dynamics: Applications of Fractional Calculus to Dynamics of Particles, Fields and Media (Springer, New York, 2011).

[5] J. Kugami, Analysis on Fractals (Cambridge University Press, 2001). 
[6] R.S. Strichartz, Differential Equations on Fractals, (Princeton Univ. Press, Princeton and Oxford, 2006).

[7] R.S. Strichartz, "Analysis on fractals", Notices of the AMS. Vol.46. No.10. (1999) 11991208.

[8] J. Harrison, "Flux across nonsmooth boundaries and fractal Gauss/Green/Stokes' theorems", Journal of Physics A. Vol.32. No.28. (1999) 5317-5328.

[9] T. Kumagai, "Recent developments of analysis on fractals", in Selected Papers on Analysis and Related Topics American Mathematical Society Translations. Vol.223. No.202. (Springer, New York, 2008) 81-96.

[10] G. Derfel, P. Grabner, F. Vogl, "Laplace operators on fractals and related functional equations", (Topical Review) Journal of Physics A. Vol.45. No.46. (2012) 463001. 34 pages. (arXiv:1206.1211)

[11] A. Carpinteri, B. Chiaia, P. Cornetti, "Static-kinematic duality and the principle of virtual work in the mechanics of fractal media", Computer Methods in Applied Mechanics and Engineering. Vol.191. No.1-2. (2001) 3-19.

[12] A. Carpinteri, P. Cornetti, "A fractional calculus approach to the description of stress and strain localization in fractal media", Chaos, Solitons and Fractals. Vol.13. No.1. (2002) 85-94.

[13] A. Carpinteri, P. Cornetti, K. M. Kolwankar, "Calculation of the tensile and flexural strength of disordered materials using fractional calculus", Chaos, Solitons and Fractals. Vol.21. No.3. (2004) 623-632.

[14] A. Carpinteri, B. Chiaia, P. Cornetti, "A disordered microstructure material model based on fractal geometry and fractional calculus", Zeitschrift für Angewandte Mathematik und Mechanik. Vol.84. No.2. (2004) 128-135.

[15] A. Carpinteri, P. Cornetti, A. Sapora, M. Di Paola, M. Zingales, "Fractional calculus in solid mechanics: Local versus non-local approach", Physica Scripta. Vol.T136. (2009) 14003.

[16] A. Carpinteri, P. Cornetti, A. Sapora, "Static-kinematic fractional operators for fractal and non-local solids", Zeitschrift für Angewandte Mathematik und Mechanik. Vol.89. No.3. (2009) 207-217.

[17] X.-J. Yang, H.M. Srivastava, J.-H. Hed, D. Baleanu, "Cantor-type cylindrical-coordinate method for differential equations with local fractional derivatives", Physics Letters A. Vol.377. No.28-30 (2013) 1696-1700.

[18] X.-J. Yang, D. Baleanu, J.A. Tenreiro Machado, "Systems of Navier-Stokes equations on Cantor sets", Mathematical Problems in Engineering. Vol.2013. (2013) 769724. 8 pages.

[19] X.-J. Yang, Advanced Local Fractional Calculus and Its Applications (World Science Publisher, New York, 2012).

[20] V.E. Tarasov, "Continuous medium model for fractal media", Physics Letters A. Vol.336. N.2-3. (2005) 167-174. (arXiv:cond-mat/0506137) 
[21] V.E. Tarasov, "Fractional hydrodynamic equations for fractal media", Annals of Physics. Vol.318. No.2. (2005) 286-307. (arXiv:physics/0602096)

[22] V.E. Tarasov, "Dynamics of fractal solid", International Journal of Modern Physics B. Vol.19. No.27. (2005) 4103-4114. (arXiv:0710.0787)

[23] V.E. Tarasov, "Wave equation for fractal solid string", Modern Physics Letters B. Vol.19. No.15. (2005) 721-728. (arXiv:physics/0605006)

[24] M. Ostoja-Starzewski, J. Li, "Fractal materials, beams and fracture mechanics", Zeitschrift für Angewandte Mathematik und Physik. Vol.60. No.6. (2009) 1194-1205.

[25] J. Li, M. Ostoja-Starzewski, "Fractal solids, product measures and fractional wave equations", Proceedings of the Royal Society A. Vol.465. No.2108. (2009) 2521-2536; and J. Li, M. Ostoja-Starzewski, "Correction to Li and Ostoja-Starzewski 465 (2108) 2521", Proceedings of the Royal Society A. Vol.467. No.2128. (2011) 1214 (1 page).

[26] P.N. Demmie, M. Ostoja-Starzewski, "Waves in fractal media", Journal of Elasticity. Vol.104. No.1-2. (2011) 187-204.

[27] J. Li, M. Ostoja-Starzewski, "Micropolar continuum mechanics of fractal media", International Journal of Engineering Science. Vol.49. No.12. (2011) 1302-1310.

[28] M. Ostoja-Starzewski, J. Li, H. Joumaa, P.N. Demmie, "From fractal media to continuum mechanics", Zeitschrift für Angewandte Mathematik und Mechanik. (Journal of Applied Mathematics and Mechanics). Vol.94. No.5. (2014) 373-401.

[29] A.S. Balankin, B.E. Elizarraraz, "Hydrodynamics of fractal continuum flow", Physical Review E. Vol.85. No.2. (2012) 025302. 6 pages.

[30] A.S. Balankin, B.E. Elizarraraz, "Map of fluid flow in fractal porous medium into fractal continuum flow", Physical Review E. Vol.85. No.5. (2012) 056314. 21 pages.

[31] J. Li, M. Ostoja-Starzewski, "Comment on "Hydrodynamics of fractal continuum flow" and "Map of fluid flow in fractal porous medium into fractal continuum flow"." Physical Review E. Vol.88. No.5. (2013) 057001. 4 pages.

[32] A.S. Balankin, B.E. Elizarraraz, "Reply to "Comment on "Hydrodynamics of fractal continuum flow" and "Map of fluid flow in fractal porous medium into fractal continuum flow"." Physical Review E. Vol.88. No.5. (2013) 057002. 6 pages.

[33] J.C. Collins, Renormalization (Cambridge University Press, Cambridge, 1984).

[34] F.H. Stillinger, "Axiomatic basis for spaces with noninteger dimensions", Journal of Mathematical Physics. Vol.18. No.6. (1977) 1224-1234.

[35] C. Palmer, P.N. Stavrinou, "Equations of motion in a non-integer-dimensional space", Journal of Physics A. Vol.37. No.27. (2004) 6987-7003.

[36] K.G. Wilson, M.E. Fisher, "Critical exponents in 3.99 dimensions", Physical Review Letters. Vol.28. No.4. (1972) 240-243.

[37] K.G. Wilson, J. Kogut, "The renormalization group and the $\epsilon$ expansion", Physics Reports. Vol.12. No.2. (1974) 75-199.

[38] G. 't Hooft, M. Veltman, "Regularization and renormalization of gauge fields", Nuclear Physics B. Vol.44. No.1. (1972) 189-213. 
[39] G. Leibbrandt, "Introduction to the technique of dimensional regularization", Reviews of Modern Physics. Vol.47. No.4. (1975) 849-876.

[40] K.G. Wilson, "Quantum field - theory models in less than 4 dimensions", Physical Review D. Vol.7. No.10. (1973) 2911-2926.

[41] X.-F. He, "Anisotropy and isotropy: A model of fraction-dimensional space", Solid State Communications. Vol.75. No.2. (1990) 111-114.

[42] X.-F. He, "Fractional dimensionality and fractional derivative spectra of interband optical transitions", Physical Review B. Vol.42. No.18. (1990) 11751-11756.

[43] X.-F. He, "Excitons in anisotropic solids: The model of fractional-dimensional space", Physical Review B. Vol.43. No.3. (1991) 2063-2069.

[44] A. Thilagam, "Exciton-phonon interaction in fractional dimensional space", Physical Review B. Vol.56. No.15. (1997) 9798-9804.

[45] A. Matos-Abiague, "Deformation of quantum mechanics in fractional-dimensional space", Journal of Physics A. Vol.34. No.49. (2001) 11059-11068. (arXiv:quant-ph/0107062)

[46] A. Matos-Abiague, "Bose-like oscillator in fractional-dimensional space", Journal of Physics A. Vol.34. No.14. (2001) 3125-3138.

[47] M.A. Lohe, A. Thilagam, "Quantum mechanical models in fractional dimensions", Journal of Physics A. Vol.37. No.23. (2004) 6181-6199.

[48] R. Eid, S.I. Muslih, D. Baleanu, E. Rabei, "On fractional Schrödinger equation in $\alpha-$ dimensional fractional space", Nonlinear Analysis: Real World Applications. Vol.10. No.3. (2009) 1299-1304.

[49] S.I. Muslih, "Solutions of a particle with fractional $\delta$-potential in a fractional dimensional space", International Journal of Theoretical Physics. Vol.49. No.9. (2010) 2095-2104. (arXiv:1001.4352v2)

[50] S.I. Muslih, O.P. Agrawal, "Schrodinger equation in fractional space", in Chapter 17 in Fractional Dynamics and Control (Springer, New York, 2012) Pages 209-215.

[51] G. Calcagni, G. Nardelli, M. Scalisi, "Quantum mechanics in fractional and other anomalous spacetimes", Journal of Mathematical Physics. Vol.53. No.10. (2012) 102110. (arXiv:1207.4473)

[52] J. Martins, H.V. Ribeiro, L.R. Evangelista, L.R. da Silva, E.K. Lenzi, "Fractional Schrodinger equation with noninteger dimensions", Applied Mathematics and Computation. Vol.219. No.4. (2012) 2313-2319.

[53] L.S. Lucena, L.R. da Silva, A.A. Tateishi, M.K. Lenzi, H.V. Ribeiro, E.K. Lenzi, "Solutions for a fractional diffusion equation with noninteger dimensions", Nonlinear Analysis: Real World Applications. Vol.13. No.4. (2012) 1955-1960.

[54] S.I. Muslih, D. Baleanu, "Fractional multipoles in fractional space", Nonlinear Analysis: Real World Applications. Vol.8. No.1. (2007) 198-203.

[55] D. Baleanu, A.K. Golmankhaneh, A.K. Golmankhaneh, "On electromagnetic field in fractional space", Nonlinear Analysis Real World Applications. Vol.11. No.1. (2010) 288-292. 
[56] S.I. Muslih, M. Saddallah, D. Baleanu, E. Rabei, "Lagrangian formulation of Maxwell's field in fractional D dimensional space-time", Romanian Reports of Physics. Vol.55. No.7-8. (2010) 659-663.

[57] M. Zubair, M.J. Mughal, Q.A. Naqvi, "The wave equation and general plane wave solutions in fractional space", Progress in Electromagnetics Research Letters. Vol.19. (2010) 137-146.

[58] M. Zubair, M.J. Mughal, Q.A. Naqvi, "On electromagnetic wave propagation in fractional space", Nonlinear Analysis: Real World Applications. Vol.12. No.5. (2011) 2844-2850.

[59] M. Zubair, M.J. Mughal, Q.A. Naqvi, "An exact solution of the spherical wave equation in D-dimensional fractional space", Journal of Electromagnetic Waves and Applications. Vol.25. No.10. (2011) 1481-1491.

[60] M. Zubair, M.J. Mughal, Q.A. Naqvi, "An exact solution of cylindrical wave equation for electromagnetic field in fractional dimensional space", Progress in Electromagnetics Research. Vol.114. (2011) 443-455.

[61] M. Zubair, M.J. Mughal, Q.A. Naqvi, Electromagnetic Fields and Waves in Fractional Dimensional Space (Springer, Berlin, 2012).

[62] P. Moon, D.E. Spencer, "The meaning of the vector Laplacian", Journal of the Franklin Institute. Vol.256. No.6. (1953) 551-558.

[63] K. Svozil, "Quantum field theory on fractal spacetime: a new regularization method", Journal of Physics A, Vol.20. (1987) 3861-3875.

[64] V.E. Tarasov, "Fractional systems and fractional Bogoliubov hierarchy equations", Physical Review E. Vol.71. No.1. (2005) 011102. 12 pages. (arXiv:cond-mat/0505720)

[65] V.E. Tarasov, "Fractional Liouville and BBGKI equations", Journal of Physics: Conference Series. Vol.7. (2005) 17-33. (arXiv:nlin.CD/0602062)

[66] Q.Y. Zhu, M.H. Xie, J. Yang, Y. Li, "A fractal model for the coupled heat and mass transfer in porous fibrous media", International Journal of Heat and Mass Transfer. Vol.54. No.7-8. (2011) 1400-1409.

[67] E. Gabrys, M. Rybaczuk, A. Kedzia, "Blood flow simulation through fractal models of circulatory system", Chaos, Solitons and Fractals. Vol.27. No.1. (2006) 1-7.

[68] G. Jayalalitha, V. Shanthoshini Deviha, R. Uthayakumar, "Fractal model for blood flow in cardiovascular system", Computers in Biology and Medicine. Vol.38. No.6. (2008) 684-693.

[69] D.A. Benson, M.M. Meerschaert, J. Revielle, "Fractional calculus in hydrologic modeling: A numerical perspective", Advances in Water Resources. Vol.51. (2013) 479-497.

[70] S.-L. Soo, Fluid dynamics of Multiphase Systems (Blaisdell Pub. Co., 1967).

[71] R.I. Nigmatulin, Dynamics of Multiphase Media, Vol.1-2. (Hemisphere, New York, 1991).

[72] V.E. Tarasov, "Vector calculus in non-integer dimensional space and its applications to fractal media", Communications in Nonlinear Science and Numerical Simulation. Vol.20. No.2. (2015) 360-374. (arXiv:1503.02022)

[73] V.E. Tarasov, "Anisotropic fractal media by vector calculus in non-integer dimensional space", Journal of Mathematical Physics. Vol.55. No.8. (2014) 083510. (arXiv:1503.02392) 


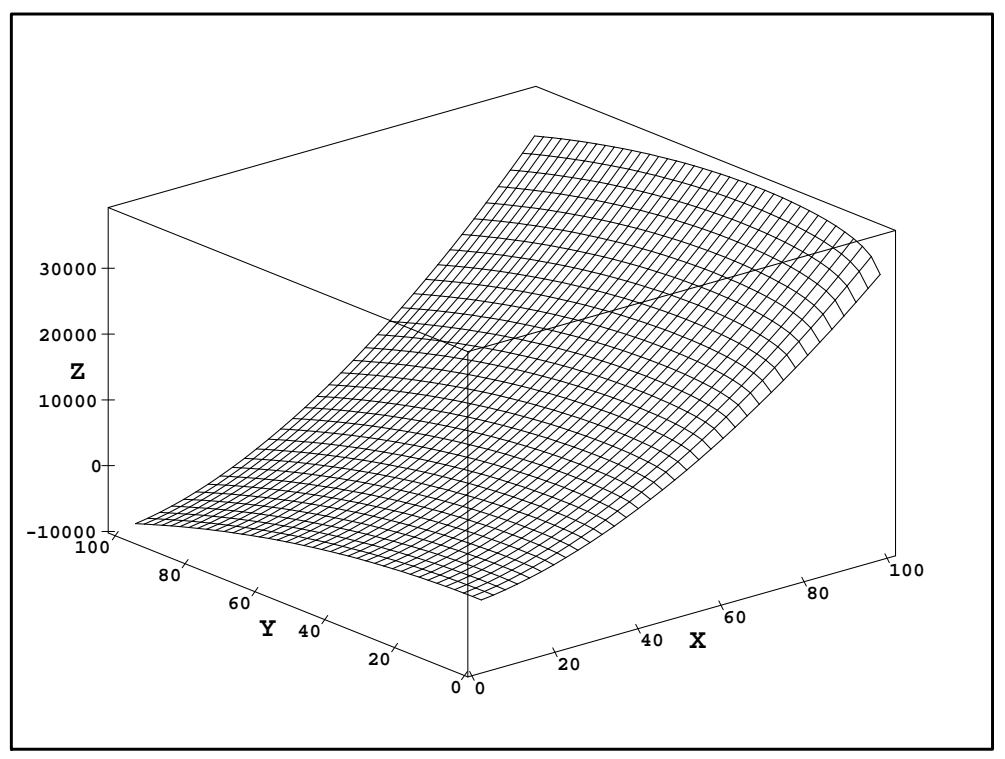

Figure 1: Plot of the velocity function $z=v(x, y)$ defined by (45) for the ranges $x \in[1 ; 100]$, $y \in[1 ; 100]$, and $D=2.9$.

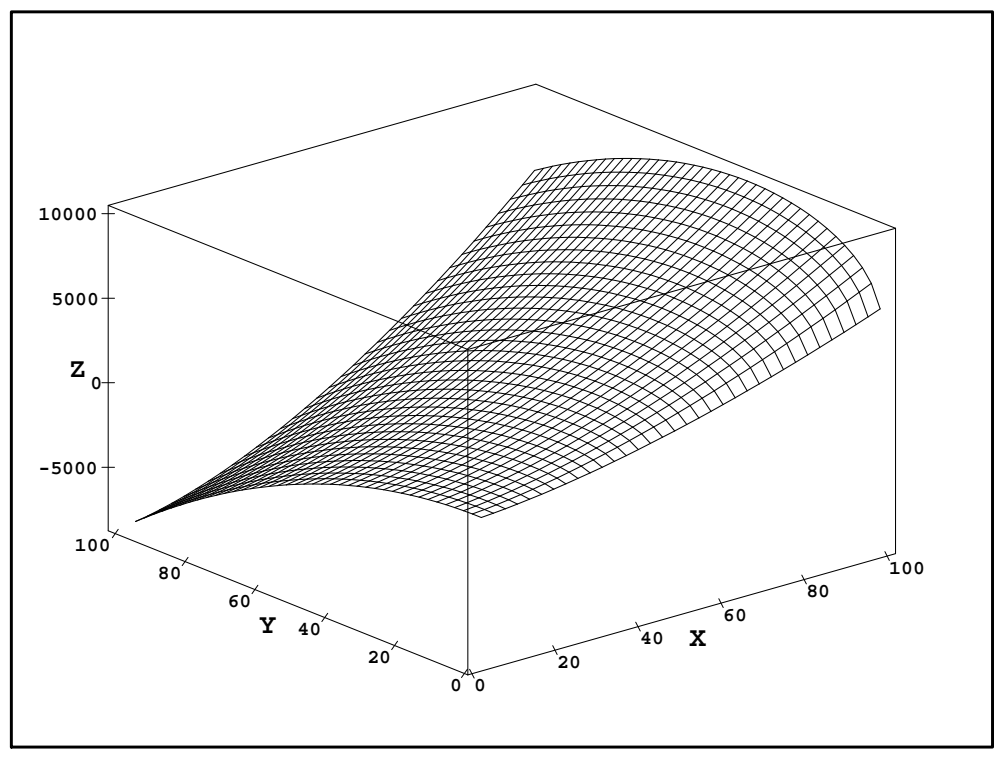

Figure 2: Plot of the velocity function $z=v(x, y)$ defined by (45) for the ranges $x \in[1 ; 100]$, $y \in[1 ; 100]$, and $D=2.7$. 


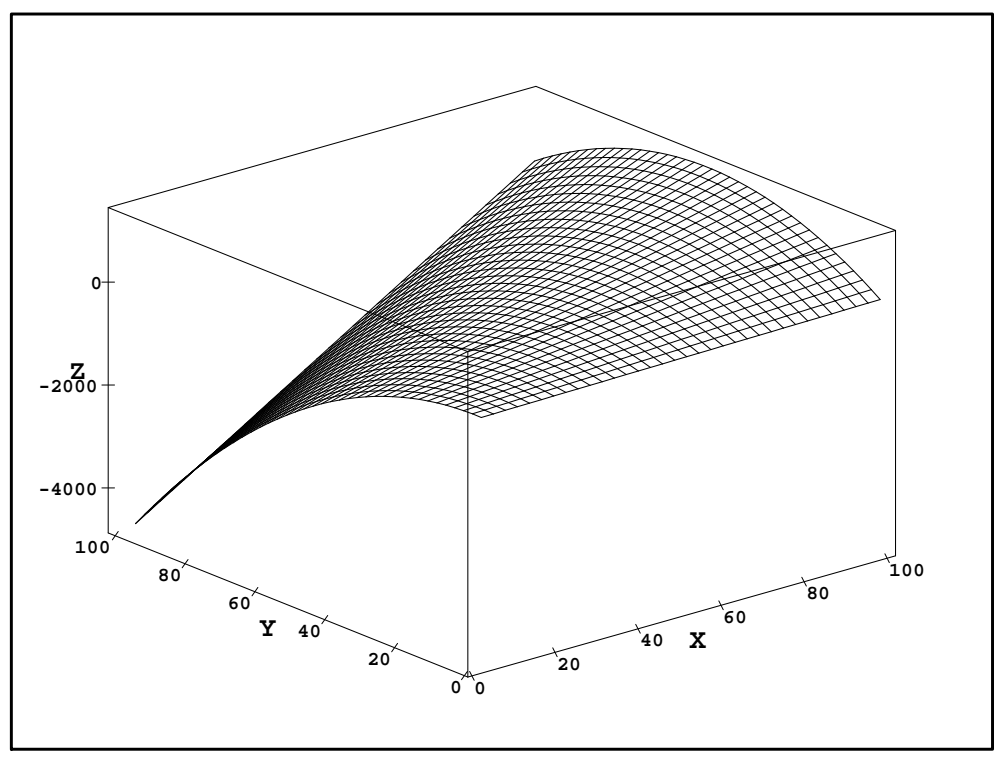

Figure 3: Plot of the velocity function $z=v(x, y)$ defined by (45) for the ranges $x \in[1 ; 100]$, $y \in[1 ; 100]$, and $D=2.0$.

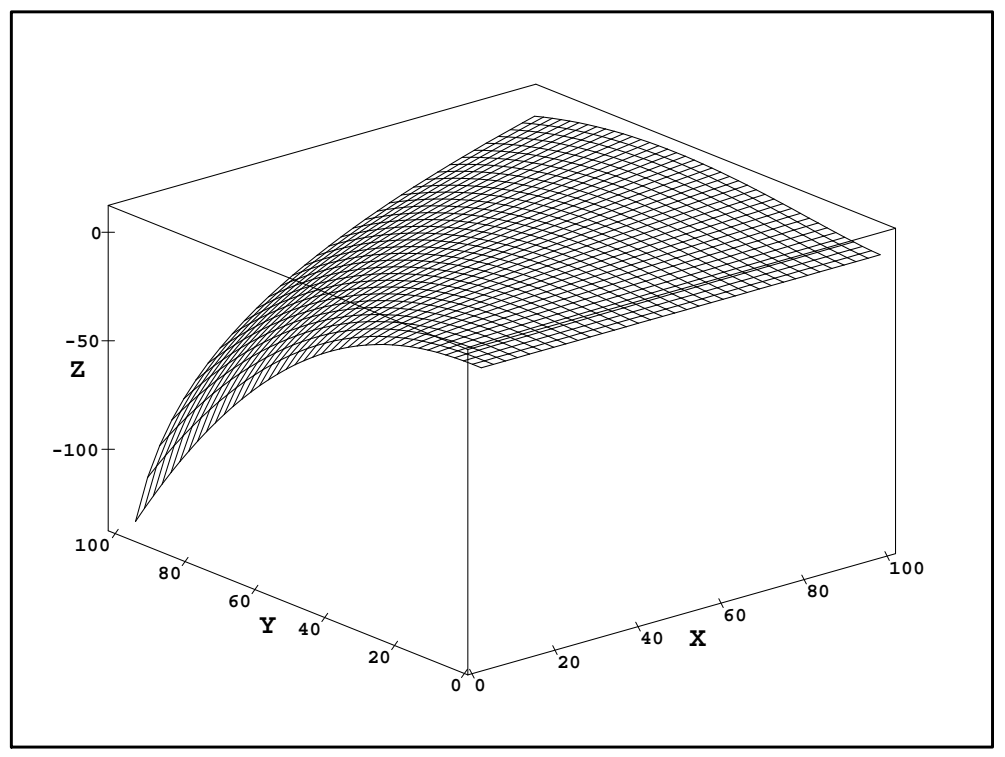

Figure 4: Plot of the velocity function $z=v(x, y)$ defined by (45) for the ranges $x \in[1 ; 100]$, $y \in[1 ; 100]$, and $D=1.1$. 


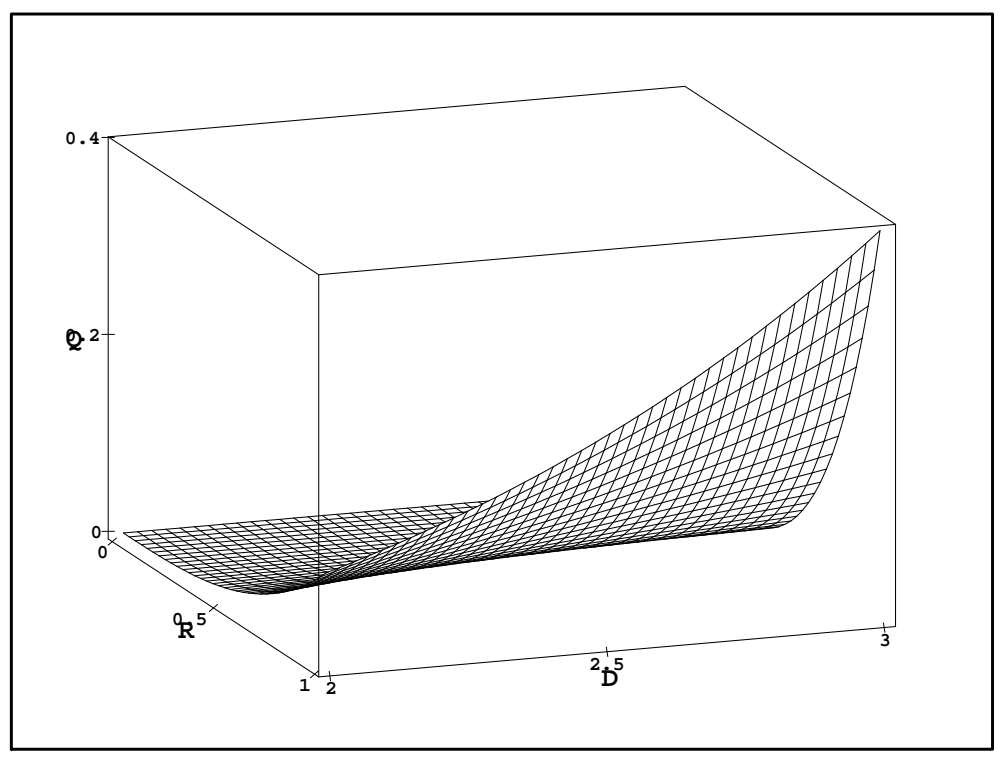

Figure 5: Plot of the discharge function $Q=Q(R, D)$ defined by (64) for the range $R \in[0,1]$ and $D \in[2,3]$.

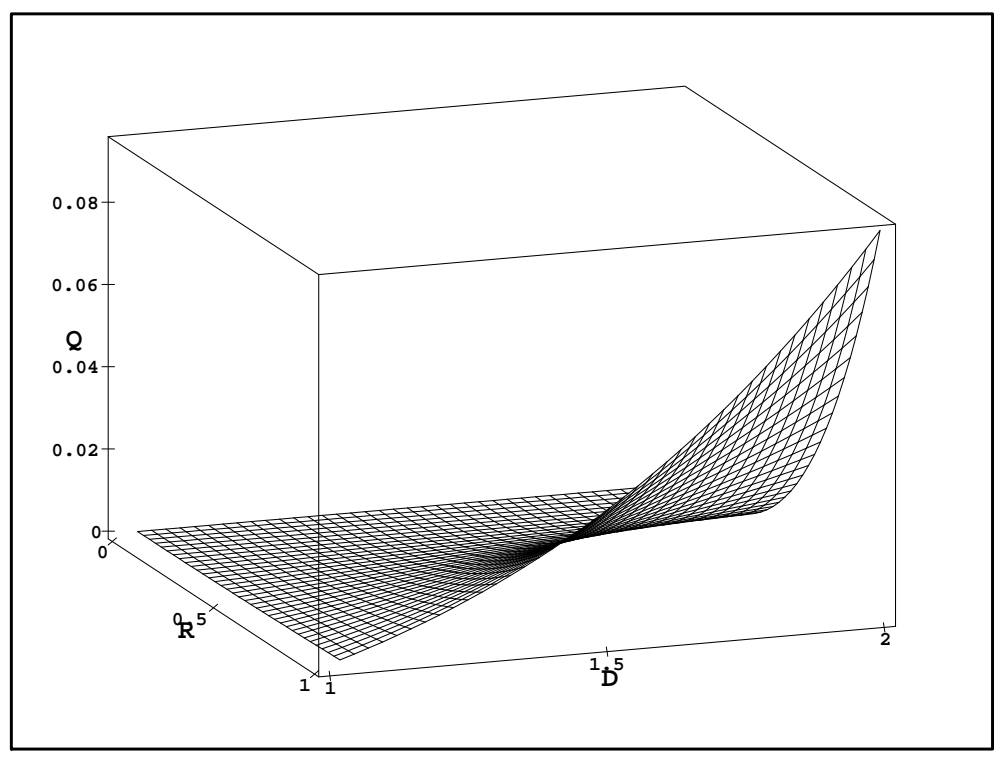

Figure 6: Plot of the discharge function $Q=Q(R, D)$ defined by (64) for the range $R \in[0,1]$ and $D \in[1,2]$. 


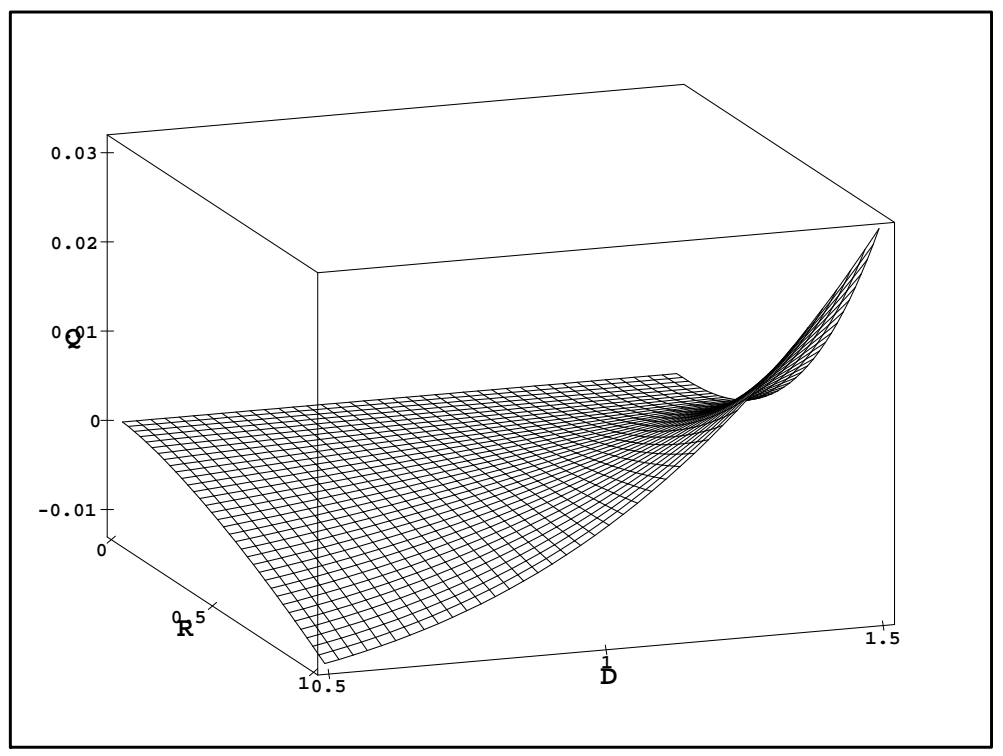

Figure 7: Plot of the discharge function $Q=Q(R, D)$ defined by (64) for the range $R \in[0,1]$ and $D \in[0.5,1.5]$.

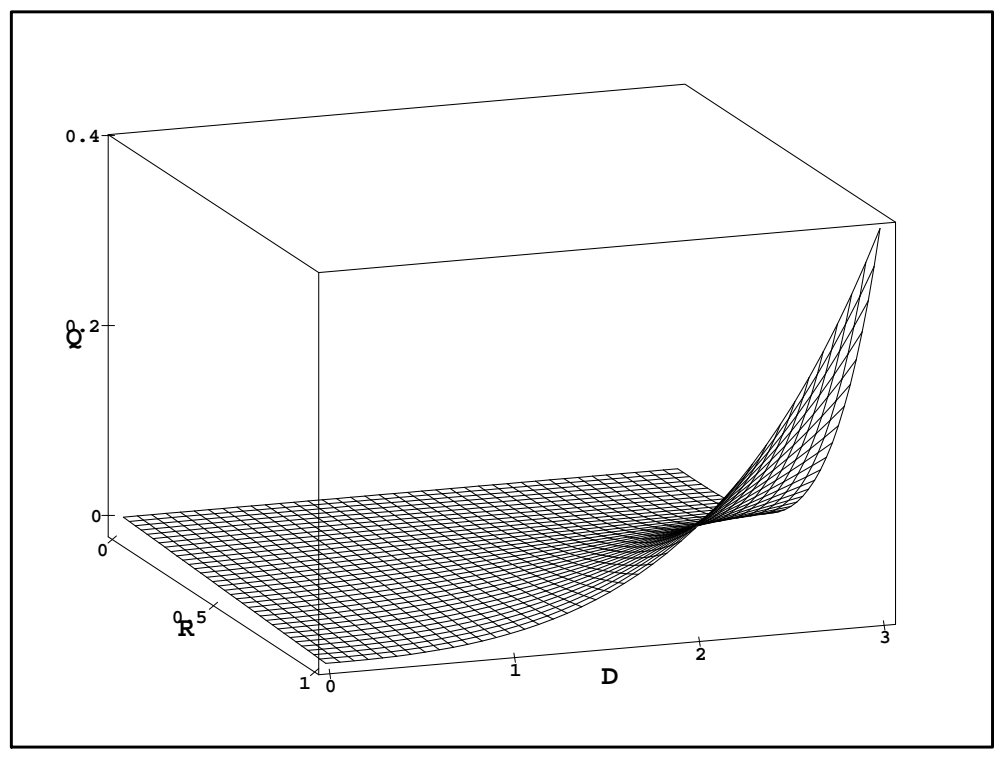

Figure 8: Plot of the discharge function $Q=Q(R, D)$ defined by (64) for the range $R \in[0,1]$ and $D \in[0,3]$. 